\title{
Permanent-magnet helicon sources and arrays: a new type of rf plasma
}

Francis F. Chen ${ }^{\text {a) }}$ and Humberto Torreblanca ${ }^{\text {b) }}$

University of California, Los Angeles, California 90095-1594

Helicon discharges are known for their ability to produce high densities of partially ionized plasma, their efficiency arising from an unusual mechanism of RF coupling. However, the requirement of a dc magnetic field has prevented their wide acceptance in industry. The use of permanent magnets greatly simplifies helicon sources, and arrays of small sources extends the use of helicons to the processing of large substrates. An eight-tube array was designed and constructed, and its density uniformity measured in a $53 \times 165 \mathrm{~cm}$ chamber. Three innovations involved a) the remote field of ring magnets, b) a low-field density peak, and c) rf power distribution. High-density plasmas uniform over large areas requires compatibility in all three areas.

\section{Introduction}

Helicon waves are whistler waves confined to a cylinder. In the lowest approximation, the frequency $\omega$ of these waves lies in the range $\Omega_{C}<<\omega_{L H}<<\omega<<\omega_{C}$, so that ion motion can be neglected, and electron motion is only their $\mathbf{E} \times \mathbf{B}$ drift. Here $\Omega_{\mathrm{c}}$ is the ion plasma frequency, $\omega_{\mathrm{LH}}$ the lower hybrid frequency, and $\omega_{\mathrm{c}}$ the electron cyclotron frequency. The dispersion relation is then simply

$$
k_{\perp} \approx\left(k_{\perp}^{2}+k_{z}^{2}\right)^{1 / 2}=e \mu_{0} \frac{\omega}{k_{z}} \frac{n_{0}}{B_{0}}
$$

where $z$ is the direction of the DC magnetic field $\mathbf{B}_{0}$, and neither mass is involved. Aside from nomenclature, Eq. (1) is identical with that of whistler waves in free space propagating at an angle to $\mathbf{B}_{0}$. However, the boundary profoundly changes the nature of the wave. As the electrons drift radially at the RF frequency, they cause oscillating charges in the cylindrical wall, if it is an insulator, or oscillating currents if it is a conductor. At the boundary, these charges or currents excite a Trivelpiece-Gould (TG) mode, which is an electron cyclotron wave in a cylinder. The TG wave is found if $\omega_{\mathrm{c}} / \omega$ is allowed to be finite. As shown in Fig. 1, the helicon

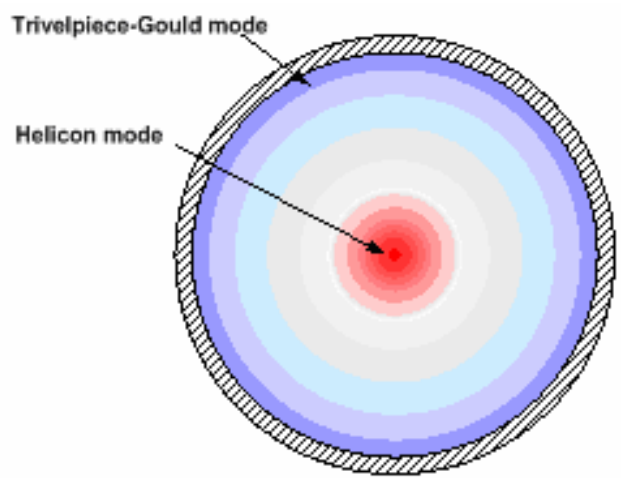

Fig. 1. Geometry of the helicon and TG waves.

\footnotetext{
a) Electronic mail: ffchen@ee.ucla.edu

b) Electronic mail: humberto.torreblanca@intel.com
} 
wave is peaked on the axis, but the TG wave is confined to a thin layer near the boundary. The TG wave is electrostatic and propagates slowly inward, depositing the rf energy into the electrons much more efficiently than does the helicon wave. The boundary has changed the electromagnetic whistler wave into a primarily electrostatic wave and greatly increased the ionization efficiency. Recent work by Lorenz et al. ${ }^{1}$ has shown that the absorption mechanism may also involve a parametric instability with the following decay scheme:

$$
\mathbf{k}_{H}=\mathbf{k}_{T G}+\mathbf{k}_{I A}
$$

Here $\mathbf{k}_{\mathrm{H}}$ is the helicon wave traveling nearly parallel to $\mathbf{B}_{0}, \mathbf{k}_{\mathrm{TG}}$ is the TG wave, and $\mathbf{k}_{\mathrm{IA}}$ is an ion acoustic wave, the last two traveling radially in almost opposite directions. The wave spectrum showed the RF drive frequency at $13 \mathrm{MHz}$ with sidebands which corresponded to the lowfrequency part of the spectrum. These sidebands widen as the power increases, showing nonlinear absorption. Thus, the science of helicons brings together four fields of plasma physics:

1. Low temperature plasmas

2. Space physics (whistler waves)

3. Magnetic fusion (DC $\mathbf{B}_{0}$ 's, RF heating, anomalous transport)

4. Laser fusion (parametric instabilities).

The anomalous transport part was investigated by Light et al. ${ }^{2}$ using $(n, \phi)$ correlation techniques.

Helicon waves were first seen in a plasma by Lehane and Thonemann ${ }^{3}$, and high-density helicon discharges were developed by R.W. Boswell ${ }^{4}$. The TG-mode absorption mechanism, replacing the Landau damping mechanism of $\mathrm{Chen}^{5}$, was promulgated by Shamrai and Taranov ${ }^{6}$. At high power, a helicon discharge contracts into a fully ionized central column with density approaching $10^{14} \mathrm{~cm}^{-3}$. There is essentially no magnetic confinement, since the ions have large Larmor radius, and the electrons can cross the field by the short-circuit effect ${ }^{7}$. At least two attempts have been made to industrialize helicon sources; one such "reactor" is the MØRI device $^{8}$ ( $\mathrm{M}=0$ Reactive Ion etcher) sold by PMT, Inc. of Chatsworth, CA. Although it performed better than existing reactors, it was not adopted by the semiconductor industry partly because of the large, heavy coaxial magnet coils and its power supply. By trial and error, the industry had developed simpler reactors not requiring a magnetic field. Plasma sources for fabrication of computer chips and flat-panel displays are already well established; but if plasma processing is needed for larger substrates, a dense, large-area source would speed up the production rate. Applications could include the following: 1) roll-to-roll processing of plastic sheets, 2) smart windows with automatic or manual opacity control, including the possibility of imbedded thin-film solar cells, 3) high-efficiency silicon solar cells, and 4) OLED displays for monitors and televisions. This paper proposes an economic way to use helicon discharges for this purpose.

\section{Array sources and antenna design}

A proof-of-principle that an array of multiple sources can produce a uniform plasma was carried out by Chen et al. ${ }^{9}$ on the 7-tube array shown in Fig. 2. A rotating probe array gave a uniformity of $\pm 3 \%$ over the surface (Fig. 3), showing that the individual sources had blended together at the substrate with no artifact of the hexagonal symmetry of the array shown in Fig. 4. The radial profiles in Fig. 5 taken with a Langmuir probe gave densities approaching $2 \times 10^{12}$ $\mathrm{cm}^{-3}$ in argon at $3 \mathrm{~kW}$ total power. Densities in the present experiment were lower because no surface magnetic confinement in the process chamber was available. 


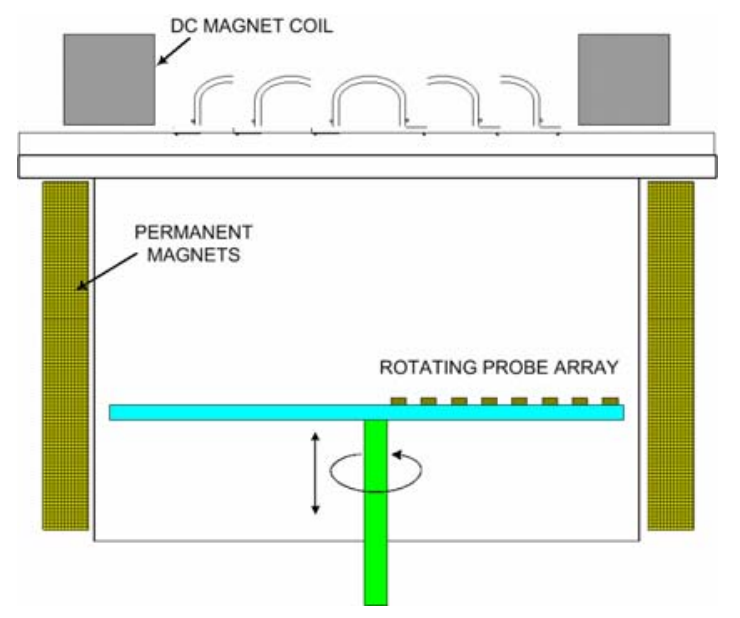

Fig. 2. The Medusa 1 distributed helicon source.

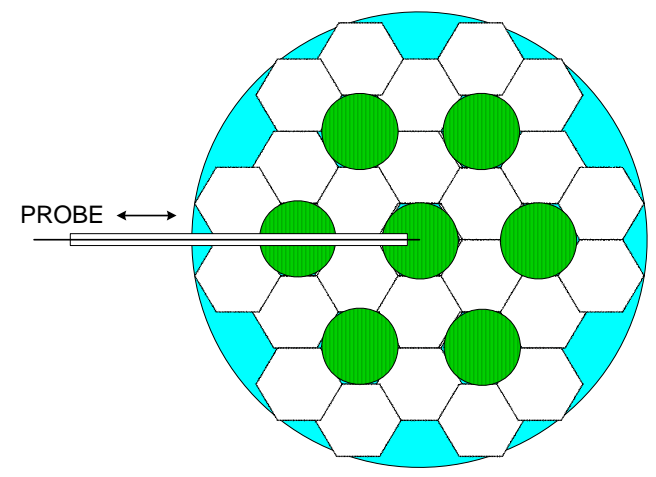

Fig. 4. Probe and source array.

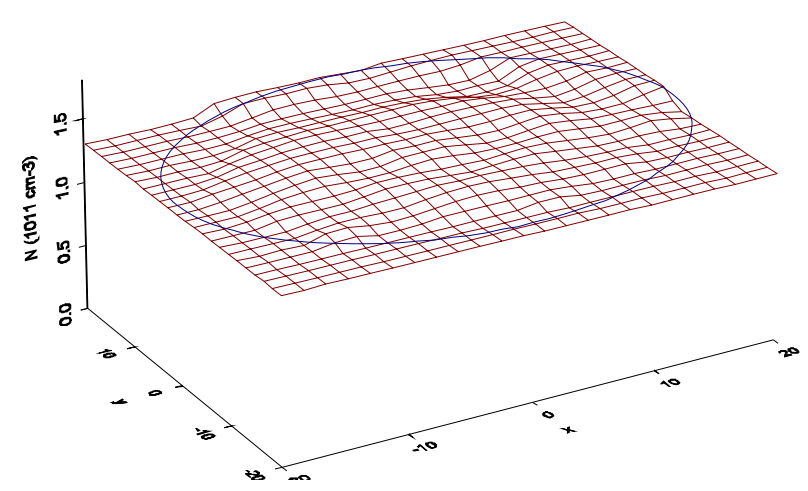

Fig. 3. 2D density profile.

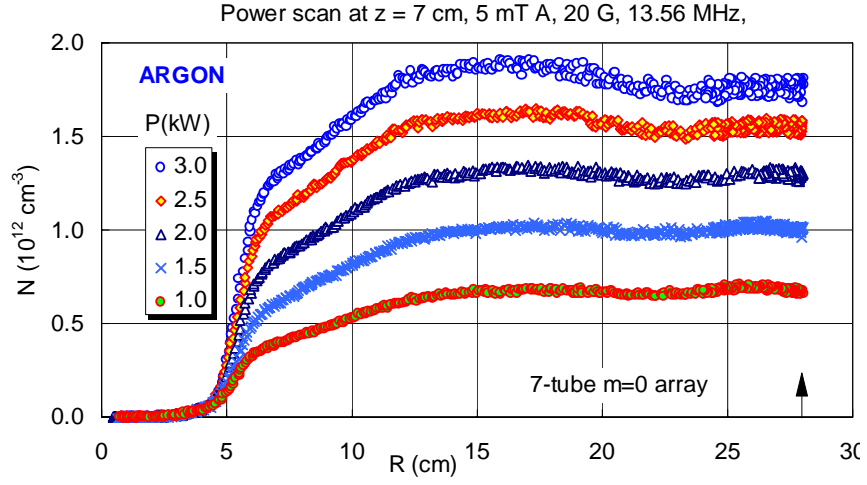

Fig. 5. Radial profiles at various RF powers.

It was necessary, however, to use a large electromagnet to create a uniform B-field. Attempts had been made earlier ${ }^{10}$ to use a small solenoid around each tube (Fig. 6), but the flaring B-field brought the plasma to the walls, and little was ejected downwards. A similar situation occurs with annular permanent magnets (PMs), as shown in Fig. 7. If the plasma is placed in the strong, uniform field region inside the magnet rings, the flare of the field will again

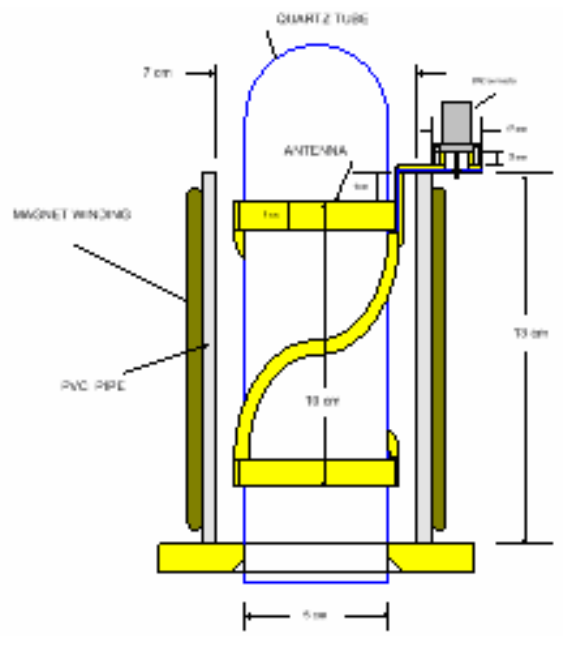

(a)

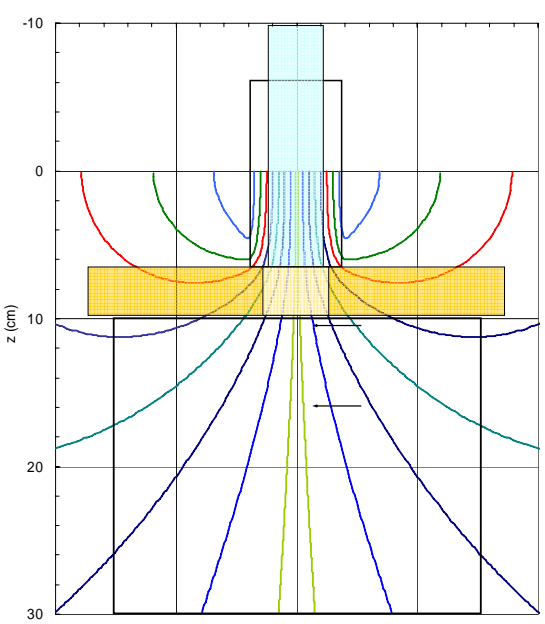

(b)

Fig. 6. (a) A helicon tube with a small solenoid ${ }^{10}$ and (b) its magnetic field lines. 


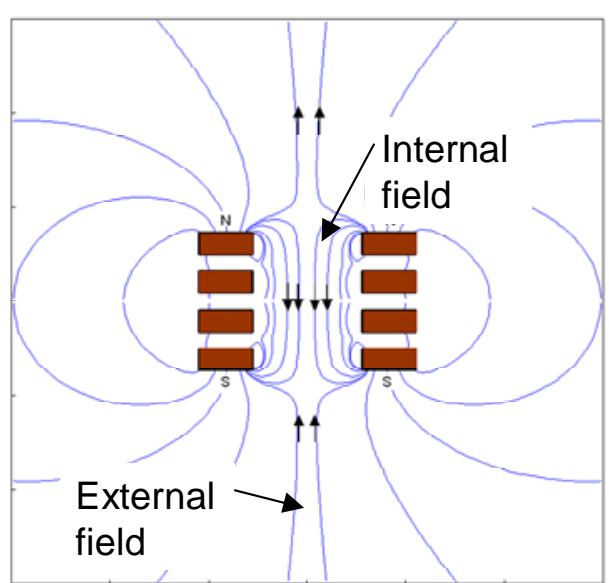

Fig. 7. Field lines of a stack of annular permanent magnets.

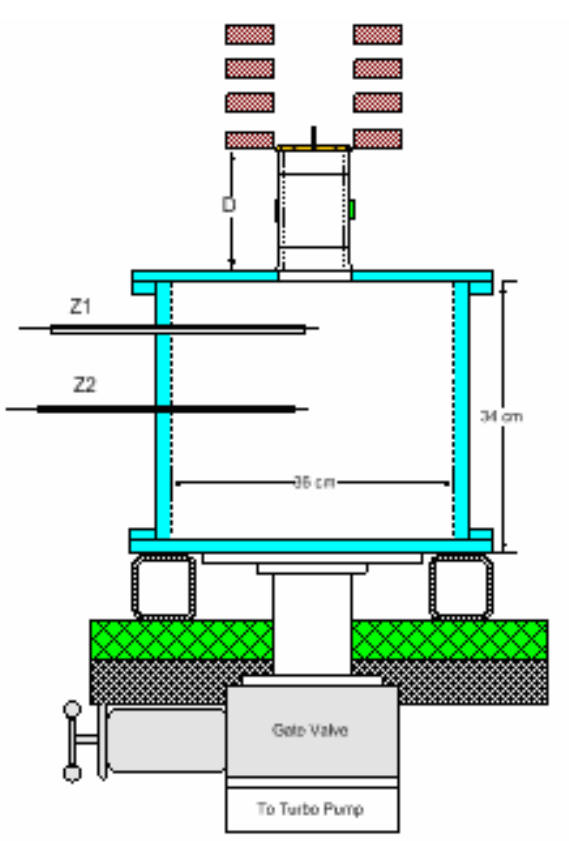

Fig. 8. Proof-of-principle apparatus.

prevent ejection of the plasma into the processing chamber. However, there is a stagnation point below the magnets at which the field reverses. If the discharge is placed in the external field, the plasma can be ejected downwards. This was proved ${ }^{11}$ in the machine of Fig. 8, where the density at the downstream probes was low until the distance $\mathrm{D}$ was increased to put the plasma well below the PM stack.

In principle, helicon waves can exist in both left- and right-hand (RH) circularly polarized versions, but only the RH wave is strongly excited. Whistlers in free space are the same way, but the reason is entirely different. For helicons, the RH wave has higher amplitude at the boundary, thus coupling strongly to the TG wave. Studies of antennas ${ }^{12}$ have shown that a helical antenna with the same helicity as the $\mathrm{RH}$ wave exhibits the best coupling. This is however not optimum for remote plasma, as shown in Fig. 9a, because a long antenna requires a long tube; and most of the plasma is lost to the walls before it is ejected. Though its coupling to the plasma is somewhat weaker, an $m=0$ loop antenna placed near the exit aperture (Fig. 9b) creates more plasma downstream. Note that the short tube includes a "skirt" to move eddy

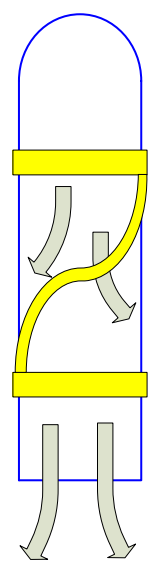

(a)

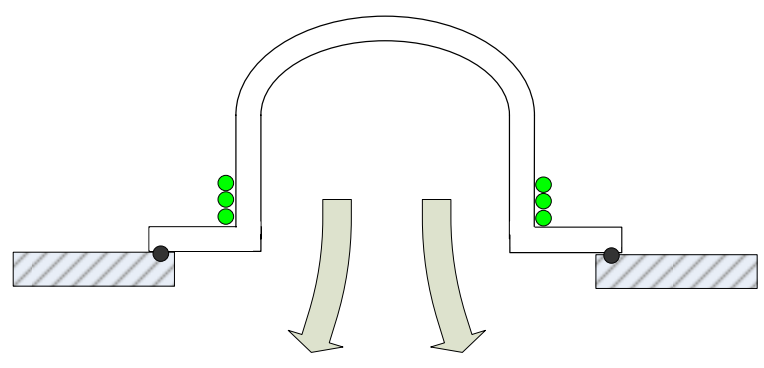

(b)

Fig. 9. Tubes with (a) an $m=1 \mathrm{RH}$ antenna and (b) an $m=0$ loop antenna. 
currents in the flange away from the antenna.

\section{Design of single tubes}

Each tube in an array of sources has to be small, since large diameter plasmas would require more diffusion distance to become uniform. A small tube, however, absorbs less power and has small plasma loading resistance $R_{\mathrm{p}}$. In that case, the stray circuit resistance $R_{\mathrm{c}}$ is a concern, and $R_{\mathrm{p}}$ has to be maximized in the design. For that we make use of the low-field peak, a density peak observed at B-fields of around 50G. This occurs for bi-directional antennas such as that in Fig. 9b and has been explained ${ }^{13}$ by the constructive interference of the backward wave reflected from the endplate with the forward wave. The resonance depends on the length $h$ of the tube, which fixes $k_{\mathrm{z}}$; but Eq. (1) shows that $k_{z}$ depends on $\omega, n$, and $B$, as well as well as $k_{\perp}$, which is fixed by the tube radius $a$. Once the desired ranges of $n$ and $B$ for the source are known, we must find the values of $\omega, a$, and $h$ for optimum $R_{\mathrm{p}}$. The standard industrial frequency of 13.56 $\mathrm{MHz}$ is preferred for $\omega$. Computations are made using the HELIC code of Arnush ${ }^{14}$, whose details cannot be given here. The code includes all linear effects in a two-fluid plasma, including antenna coupling, TG mode, collisional damping, displacement current, radial profiles, and the distance between the antenna and the endplate, which is effectively $h$. For each set of input parameters, HELIC solves a fourth-order differential equation several hundred times, once for each $k_{z}$, and sums over the antenna spectrum to obtain all the wave and absorption profiles. To permit rapid scans, the basic assumption is that $n$ and $B$ are initially uniform along $z$. Figure 10 shows a typical scan of $R_{\mathrm{p}}$ vs. $n$ and $B$, showing the low-field peak. The peak at the desired mid- $10^{12} \mathrm{~cm}^{-3}$ density occurs for $B=63 \mathrm{G}$. Here $T_{\mathrm{e}}$ enters only in the damping rate. The result of numerous scans is the small tube shown in Fig. 11. An aluminum top plate was found to be better than an insulating one. The water-cooled antenna consists of three turns of 3.2-mm diam $\mathrm{Cu}$ tubing to give the necessary inductance.

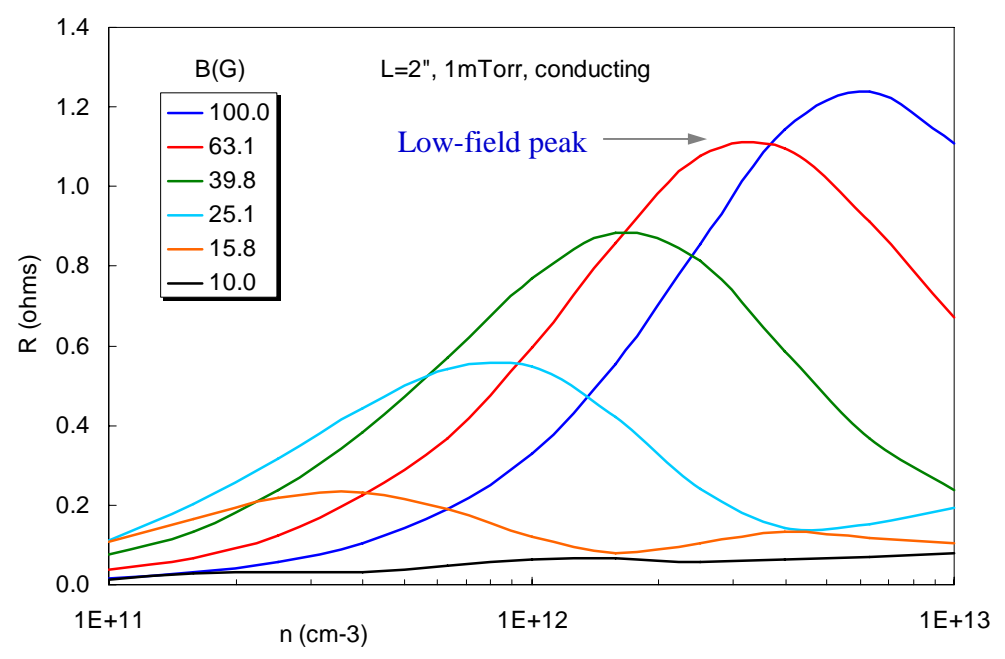

Fig. 10. Scan of $R_{\mathrm{p}}$ vs. $n$ for various B-fields for a 5-cm diam, 5-cm long tube with a close-fitted $m=0$ antenna with $1 \mathrm{mTorr}$ of argon, $13.56 \mathrm{MHz}$ rf, $T_{\mathrm{e}}=3 \mathrm{eV}$, and a wide-peaked $n(r)$.

We next designed the permanent magnet for the source by computing the field plots of annuli of different radii, thicknesses, and spacings. The field strength depended mainly on the total volume of the magnet, and the uniformity depended only weakly on the size. The final design, slightly larger than the tube, is shown in Fig. 12. NeFeB material was chosen for its high field and low cost of fabrication. The rectangles in Fig. 12b represent possible positions of the discharge tube; the B-field can be changed by simply moving the magnet up and down. Figure 
12c shows $B_{\mathrm{z}}$ in the external field, together with the position of the discharge tube when the antenna is at 63G.
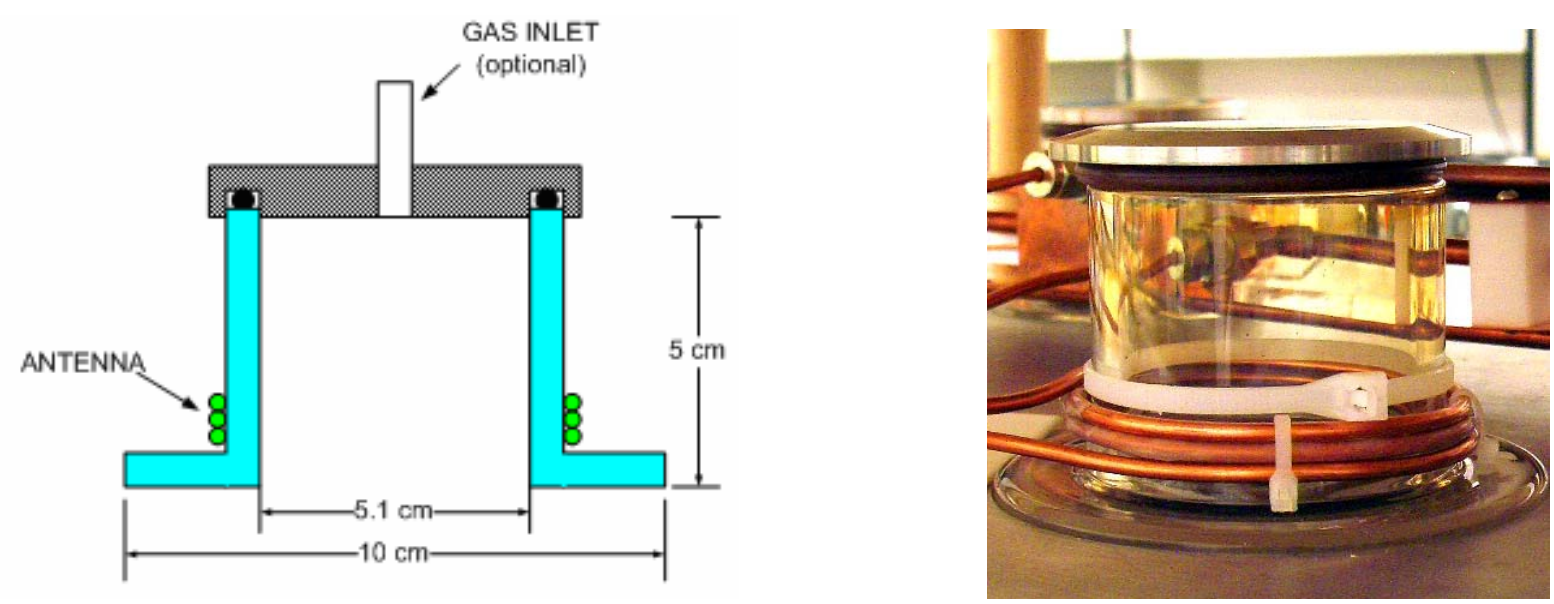

Fig. 11. Final design and appearance of discharge tube.

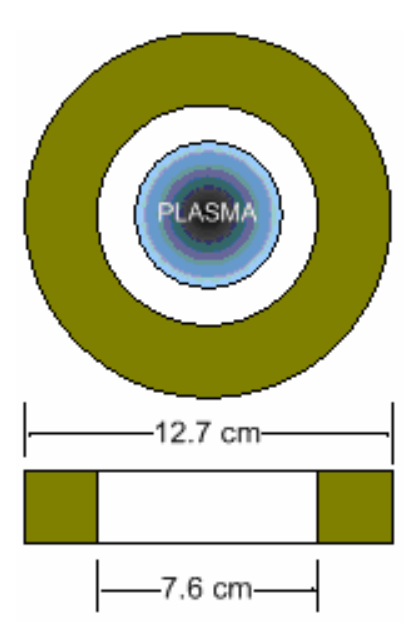

(a)

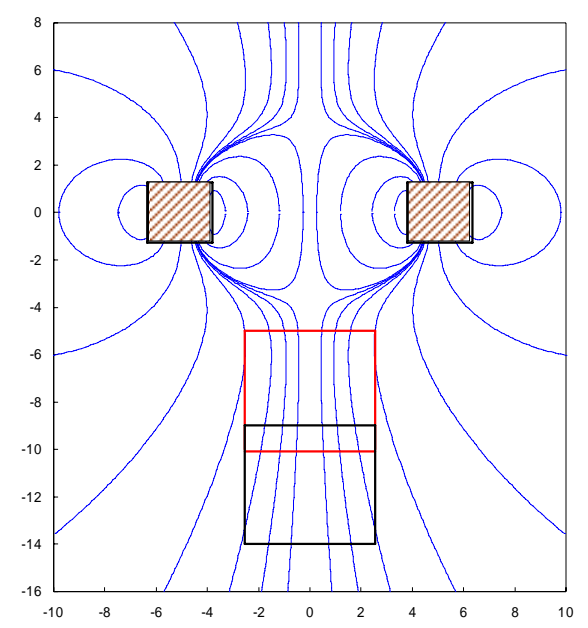

(b)

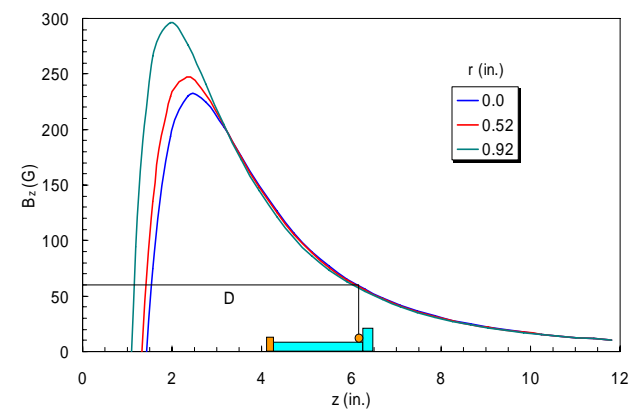

(c)

Fig. 12. NeFeB magnet design (a), field plot (b), and field strength (c). The origin in (c) is at the midplane of the antenna. The magnet is $2.54 \mathrm{~cm}$ thick.

\section{Design of source arrays}

In single-tube sources, the matching circuit can be placed close to the antenna, but large arrays require connections to the matching circuit with long cables. Small individual rf generators on each tube were rejected because of the statistical susceptibility to failure. The rf circuit, therefore, has a non-negligible stray impedance $R_{\mathrm{c}}$ relative to $R_{\mathrm{p}}$. If a power $P_{\mathrm{rf}}$ is applied to the antenna, the power $P_{\text {in }}$ that reaches the plasma is given by

$$
P_{\text {in }}=P_{r f} \frac{R_{p}}{R_{p}+R_{c}} \text {. }
$$

If $R_{\mathrm{p}}<<R_{\mathrm{c}}, P_{\text {in }}$ varies as $R_{\mathrm{p}}$, and a plot of $P_{\text {in }} v s . n$ has a shape like those in Fig.10, as shown in Fig. 13a. On the other hand, if $R_{\mathrm{p}}>>R_{\mathrm{c}}, P_{\text {in }}$ is essentially $P_{\mathrm{rf}}$, and $P_{\text {in }}$ is almost flat, as shown in 
Fig. 13b. The dashed line gives the power lost by the plasma by diffusion and radiation, and this is proportional to $n$. The equilibrium density at each $P_{\mathrm{rf}}$ is given by the intersections. Stable equilibria occur at all powers if $R_{\mathrm{p}}>>R_{\mathrm{c}}$, but only if $P_{\mathrm{rf}}$ is high enough if $R_{\mathrm{p}}<<R_{\mathrm{c}}$. The aim is to make the $R_{\mathrm{p}}$ of each tube as high as possible, and to make $R_{\mathrm{c}}$ as small as possible.

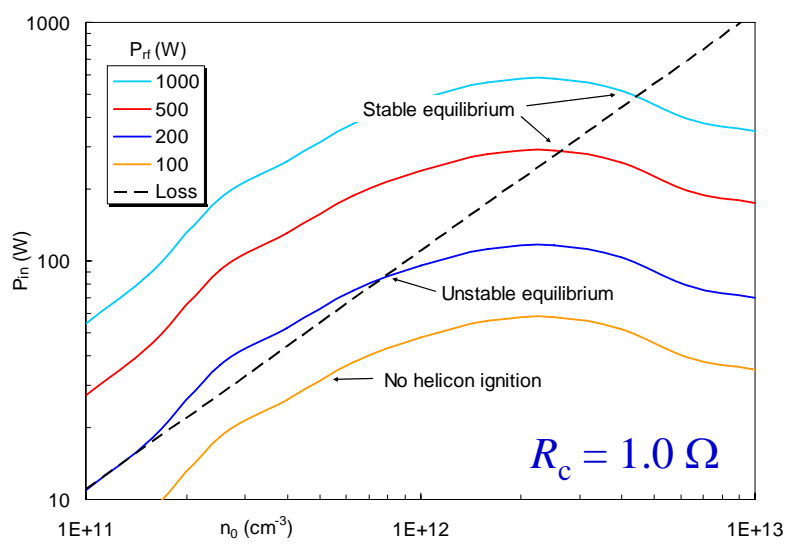

(a)

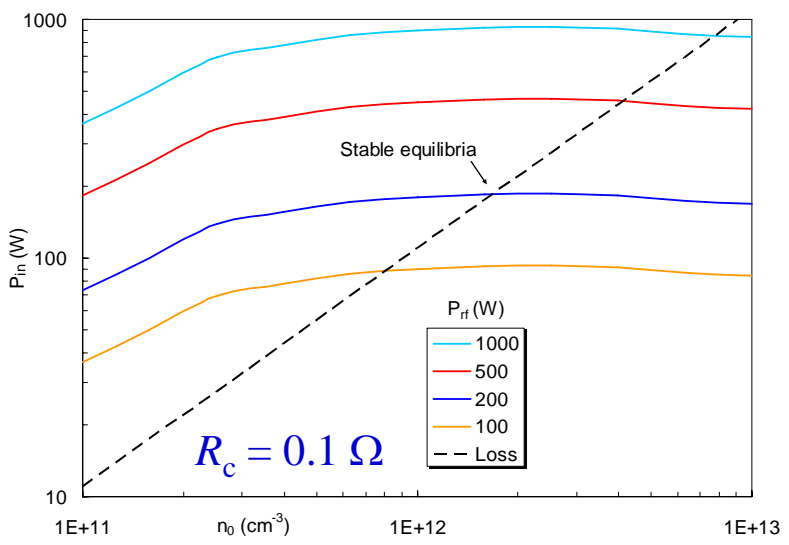

(b)

Fig. 13. Plots of $P_{\text {in }}$ Vs. $n$ and $P_{\text {rf }}$ for (a) $R_{\mathrm{p}}<<R_{\mathrm{c}}$, and (b) $R_{\mathrm{p}}>>R_{\mathrm{c}}$. Intersections with the loss line (- - -) give the equilibrium densities at each $P_{\mathrm{rf}}$.

The rf circuit is shown in Fig. 14. A standard two-capacitor matching network connects the power supply to the junction box, which distributes the power in parallel to all the tubes in the array. Each tube presents a load consisting of the antenna inductance $L$ and the plasma resistance $R\left(=R_{\mathrm{p}}\right)$. The cables affect the matching if $f \geq 13 \mathrm{MHz}$. The cable length $\mathrm{Z}_{1}$ is not critical, but the lengths $Z_{2}$ are restrictive. These cables must be of the same length, and they cannot be short because they must reach the farthest tube. Figure 15a shows that there is no

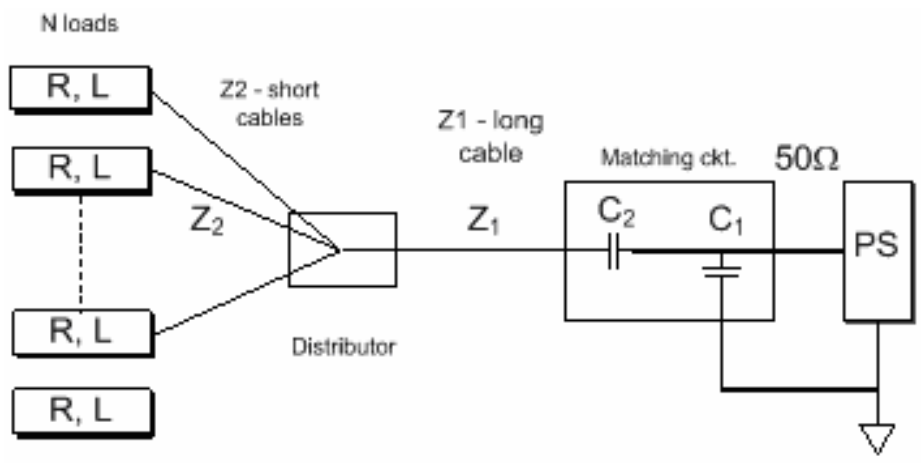

Fig. 14. Diagram of the rf circuit.

solution for the capacitor $C_{2}$ if $Z_{2}>130 \mathrm{~cm}$ in this example. For $Z_{2}=90 \mathrm{~cm}$, Fig. 15b shows that the inductance of each antenna cannot exceed $1.2 \mu \mathrm{H}$ in an array of eight tubes. An advantage of small tubes is that the number of turns in the antenna can be adjusted for matching purposes.

In CW operation, standard coaxial connectors will overheat and are subject to arcing on startup. Before the plasma ignites, the load is purely inductive, and high voltages will be applied by a power supply set for, say, 3kW when the current and voltage are $90^{\circ}$ out of phase. Consequently, all connections at the antennas and the junction box have to be soldered. In an attempt to minimize $R_{\mathrm{c}}$, we constructed the water-cooled, rectangular, $50-\Omega$ transmission line shown in Fig. 16. The inner conductor is a $6.4 \mathrm{~mm}$ diam copper pipe with soldered T-joints to each antenna. The water joints also carry the rf current. For cooling, antennas are connected in 
pairs so that no high voltage appears across the water. In a linear array, the path lengths to the antennas are not equal, and the power distribution is not uniform; but this can be corrected in rectangular or circular arrays.

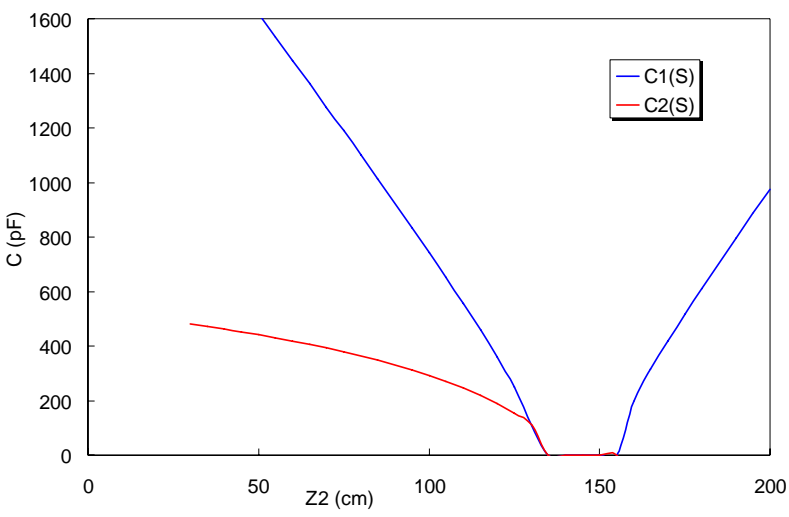

(a)

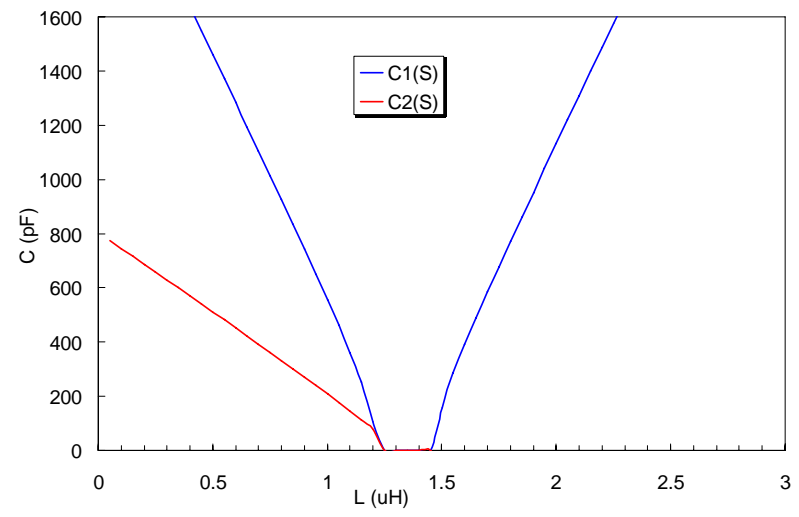

(b)

Fig. 15. Variation of $C_{1}$ and $C_{2}$ of the matching circuit in an 8-tube array for $Z_{1}=110 \mathrm{~cm}$ and (a) $L=0.8 \mu \mathrm{H} v s . Z_{2}$, and (b) $\mathrm{Z}_{2}=90 \mathrm{~cm}$ vs. $L$.

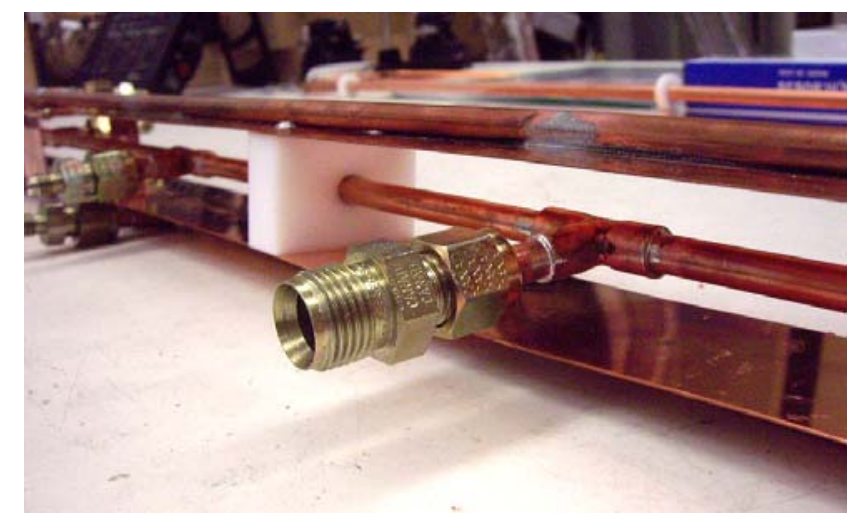

Fig. 16. Section of a 50- $\Omega$ rectangular transmission line with antenna tap-offs.

\section{Design and testing of an eight-tube array}

The chamber shown in Fig. 17 was designed to accommodate eight tubes of the design given in Sec. III in either a compact configuration (the eight centered tubes) or a staggered configuration (the darker tubes). The latter arrangement is for a substrate that moves in the $y$ direction under both rows. The spacing of the tubes is adjusted to give $\pm 2 \%$ uniformity based on the $n(r)$ measured from a single tube. In the side view of Fig. 18, the magnets are split in half so that they can be held by an aluminum sheet. Their heights can be adjusted to vary the B-field. Note that although the array can be made as large as necessary and the chamber can be as deep as necessary, the source itself requires only $15 \mathrm{~cm}$ of vertical space. In the experiment, since the magnets have to be moved to different positions, they were held in a wooden tray with movable partitions and rods to hold the magnets in the same plane so that they would not flip over onto each other. Measurements were made with Langmuir probes in the four ports, two under a row of tubes, and two between rows. There was also a 15-probe array to measure the 2D density uniformity at any level below the source. Figure 19 shows the 8-tube array source operating in 
the staggered configuration and powered by the rectangular transmission line. With quartz tubes the source can run in steady state.

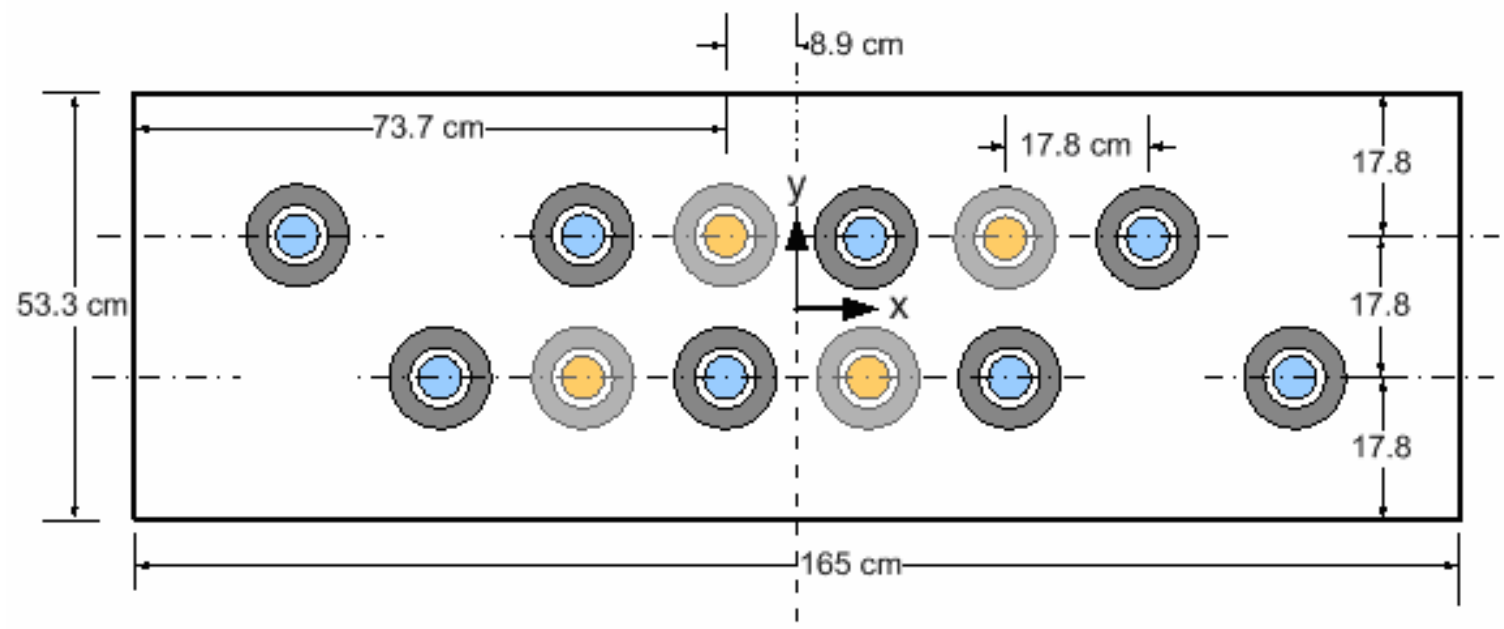

Fig. 17. Top view of experimental chamber.

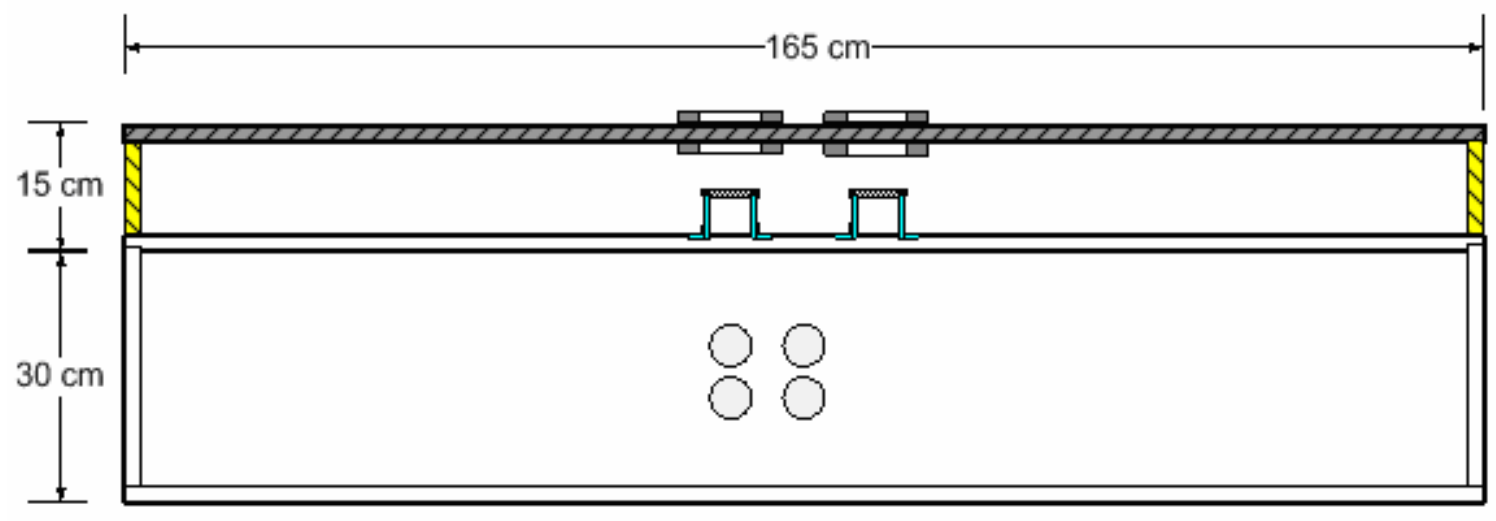

Fig. 18. Side view of experimental chamber.

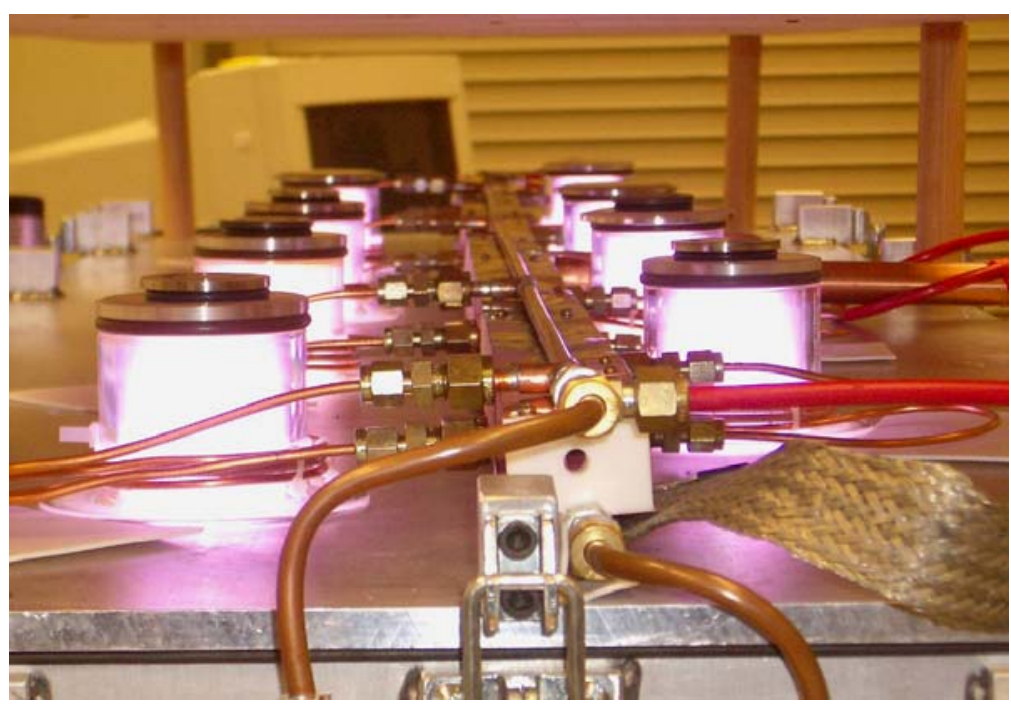

Fig. 19. The Medusa 2 test source in steady state operation. 
We give three examples of density measurements in this device. Figure 20 shows a density profile in the $y$ direction in the compact configuration with the probe going between tubes. The positions of the two rows are shown, as well as the walls of the chamber. The density is uniform in the region of the sources but falls to a low value at the walls because there is no confinement with surface magnets was used. For this reason and because a larger area is covered, the density is lower than in the previous experiment of Fig. 5.

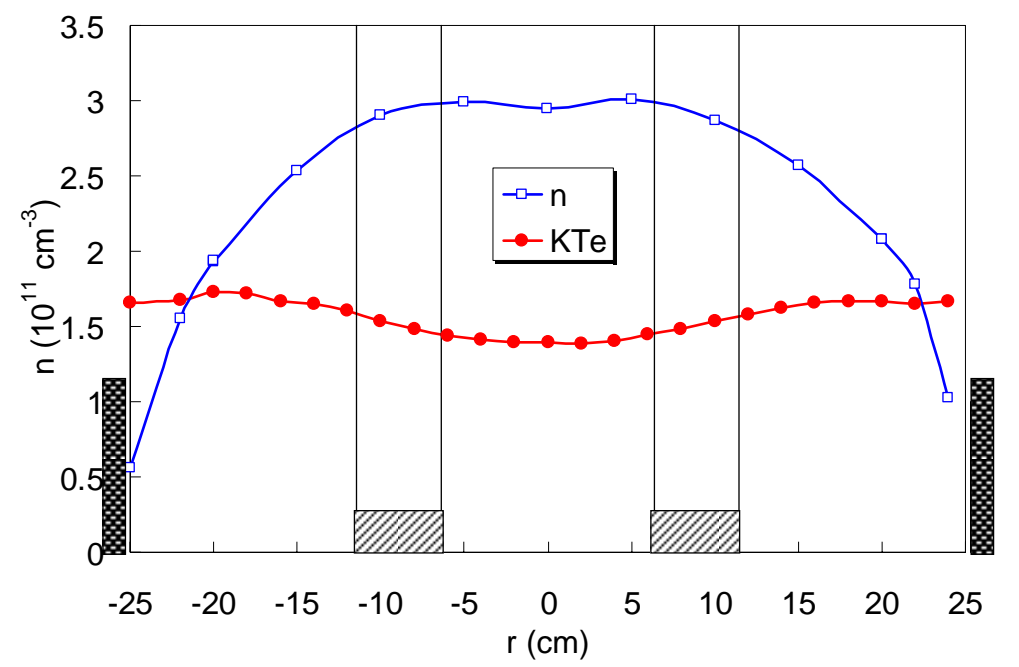

Fig. 20. "Radial” density and $T_{\mathrm{e}}$ profiles $19 \mathrm{~cm}$ below the source at $3 \mathrm{~kW}$ and $15 \mathrm{mTorr}$ of Ar.

Figure 21 shows $n(x)$ along the chamber, $19 \mathrm{~cm}$ below the source, taken with three of the 15 probes in the bottom probe array. In the staggered configuration, there are irregularities at $y=$ \pm 3.5 in. under the rows of tubes, but the density is very uniform at $y=0$ between the rows. Figure 22 is a similar scan with the compact configuration. The profiles are smoother, but $n$ falls off at the edges because of the smaller size of the array. In changing from the staggered to the compact configuration, the magnet height had to be increased because the B-field in each tube was increased by the stray field from neighboring magnets.

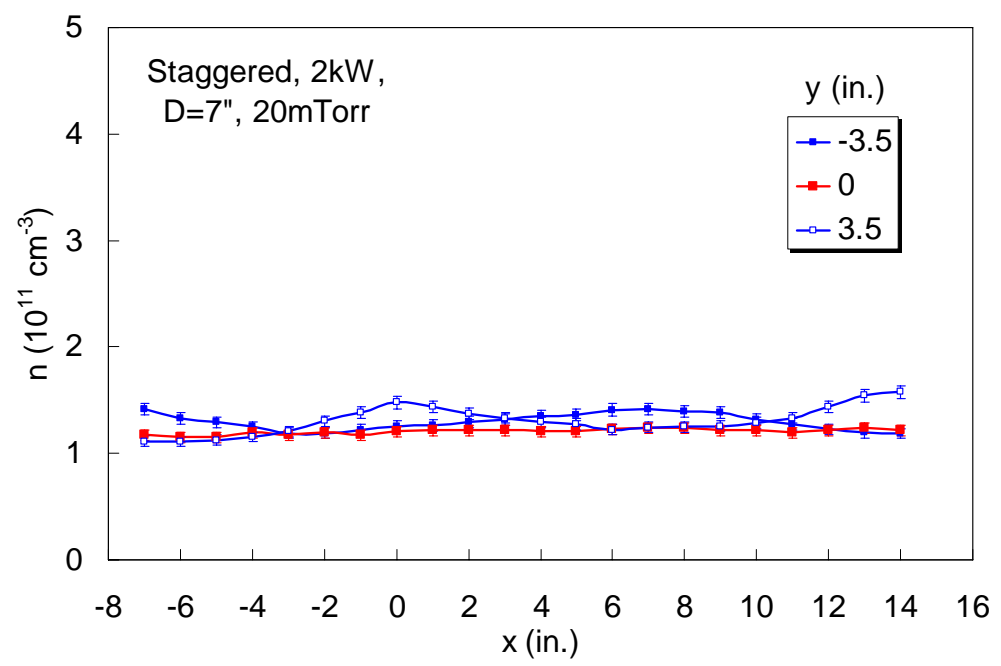

Fig. 21. Density profiles along the chamber in the staggered configuration. 


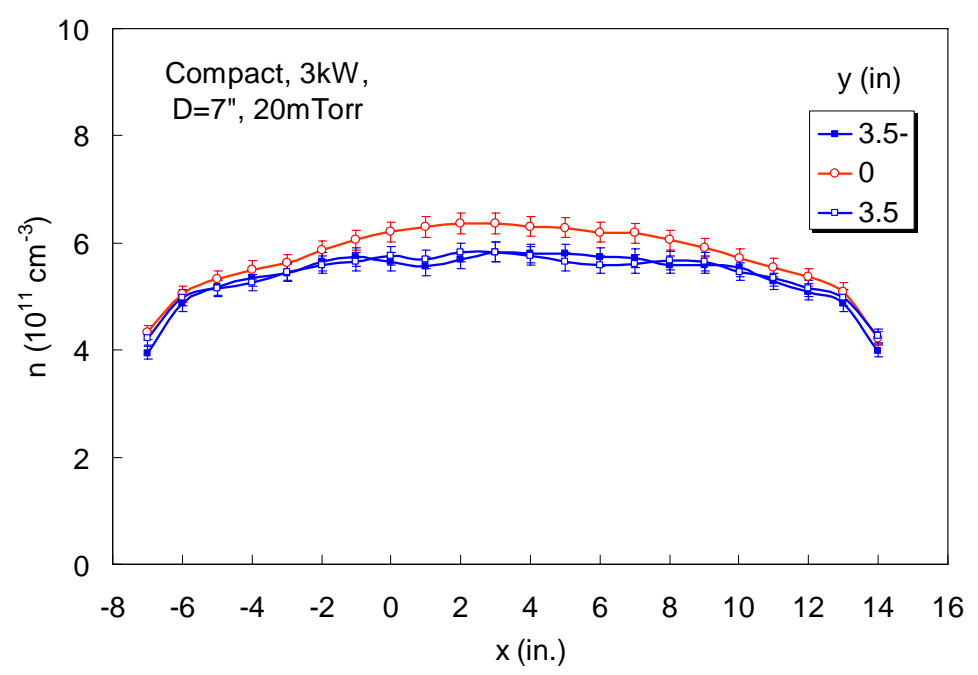

Fig. 22. Density profiles along the chamber in the compact configuration.

\section{Conclusions}

The industrial viability of high-density helicon rf sources has been improved by employing permanent magnets and making use of the low-field density peak. Large area substrates can be covered uniformly with plasma produced by arrays of small sources. The interrelations between the discharge tube, the magnet, and the rf circuit are critical in the design. This concept was proved experimentally in an 8-tube array built to run in steady state.

This project initially funded by the National Science Foundation. Subsequent support was received from Sencera LLC and the Institute of Nuclear Energy Research, Taiwan. Hiden Analytical, Ltd., provided in-kind diagnostics support. 


\section{REFERENCES}

${ }^{1}$ B. Lorenz, M. Krämer, V.L. Selenin, and Yu.M. Aliev, Plasma Sources Sci.Technol. 14, 623 (2005).

${ }^{2}$ M. Light, F.F. Chen, and P.L. Colestock, Phys. Plasmas 8, 4675 (2001).

${ }^{3}$ J.A. Lehane and P.C. Thonemann, Proc. Phys. Soc. 85, 301 (1965).

${ }^{4}$ R.W. Boswell, Plasma Phys. Control. Fusion 26, 1147 (1984).

${ }^{5}$ F.F. Chen, Plasma Phys. Control. Fusion 33, 339 (1991).

${ }^{6}$ K.P. Shamrai and V.B. Taranov, Plasma Sources Sci. Technol. 5, 474 (1996).

${ }^{7}$ A. Simon, Phys. Rev. 98, 317 (1955).

${ }^{8}$ G.R. Tynan, A.D. Bailey III, G.A. Campbell, R. Charatan, A. de Chambrier, G. Gibson, D.J. Hemker, K. Jones, A. Kuthi, C. Lee, T. Shoji, and M. Wilcoxson, J. Vac. Sci. Technol. A 15, 2885 (1997).

${ }^{9}$ F.F. Chen, J.D. Evans, and G.R. Tynan, Plasma Sources Sci. Technol. 10, 236 (2001).

${ }^{10}$ F.F. Chen, X. Jiang, and J.D. Evans, J. Vac. Sci. Technol. A 8, 2108 (2000).

${ }^{11}$ F.F. Chen and H. Torreblanca, Plasma Phys. Control. Fusion 49, A81 (2007).

${ }^{12}$ D.G. Miljak and F.F. Chen, Plasma Sources Sci. Technol. 7, 61 (1998).

${ }^{13}$ F.F. Chen, Phys. Plasmas 10, 2586 (2003).

${ }^{14}$ D. Arnush, Phys. Plasmas 7, 3042 (2000). 